\title{
High-Resolving Mass Analyzers
}

\author{
H. Wollnik
}

Dept. Chemistry \& Biochemistry, New Mexico State University, Las Cruces, USA

\begin{abstract}
First high-resolving mass analyzers were built $\approx 80$ years ago as sector field systems, well reproducible ones, however, only much later. Besides these sector-field systems there are three other types of mass analyzers: (1) Penning trap mass analyzers, have achieved the highest resolving powers, but require big technological efforts. (2) Time-of-flight mass analyzers have become the most versatile systems, while high performing multi-reflection time-of-flight systems have only started to be used. (3) Fourier Transform and Orbitrap mass analyzers have achieved spectacularly high mass resolving powers, but are also technically demanding and difficult to build systems.
\end{abstract}

\section{Introduction}

To separate ions of masses $m_{0}$ and $m_{0}\left(1+\delta_{m}\right)$ for small values of $\delta m$ has been the goal of mass analyzers, since the mass analysis of ions started about 100 years ago [1]. Herein the capability of mass analyzers is described as its mass resolving power $R_{m}$, which is the inverse of the smallest resolvable $\left(\delta_{m}\right)_{\text {min }}$. There are two main applications that require large mass resolving powers $R_{m}$.

- The precise determination of the masses of atoms provides direct information on nuclear binding energies. This is most important for the understanding of the rapid neutron capture and decay of nuclei in supernova star explosions, where heavy elements are bred [2].

- The precise determination of the masses of molecules allows to identify complex molecules and to determine their composition in medical, pharmacological, biological and/or environmental investigations [3].

\section{Angle- and Energy-Focusing Sector-Field Mass-Analyzers}

First successful mass analyzers were built by using electromagnetic sector field arrangements that can analyze ions of equal charge $q$ and masses $m=m_{0}\left(1 \pm \delta_{m}\right)$ while their energies $K\left(1 \pm \delta_{K}\right)$ vary by $\delta_{K} \leq 1 \%$. Using magnetic sector fields of strength $B_{0}$ and electrostatic sector fields of strength $E_{0}$, in which ions move along radii $\rho_{B}=\sqrt{2 K m} / q B_{0}$ and $\rho_{E}=K / q E_{0}$, respectively, it is possible to combine them so [4] that the ion arrival positions at the ion detector are independent of the energy spread $\pm K_{0} \delta_{K}$ of the ions as well as of the initial angles of inclinations $\alpha \leq \alpha_{0}$ of the ion trajectories. Such mass analyzers feature a pure mass dispersion $[1,4]$ as long as $\left(\delta_{K} \ll 1\right)$ and $\left(\alpha_{0} \ll 1\right)$.

Though such mass analyzers commonly achieve mass resolving powers of a few 10,000, higher mass resolving powers were reached, when enough effort was spent especially in the correction of image aberrations, i.e. the terms proportional to $\alpha_{0}^{2}, \delta_{K}^{2}, \alpha_{0}^{3}, \delta_{K}^{3} \ldots$. Mass analyzers that achieve simultaneously high mass resolving powers and high ion transmissions could only be build, when the complex ion motion through the fringing fields of magnetic and electric sector fields was understood [4] and when, additionally, the mechanical precision of the systems became sufficiently good.

Sector-field mass analyzers were usually built as mass spectrographs [1,4], in which ions of different masses were recorded simultaneously in a position sensitive detector. However, they can also be used as spectrometers [5] in which the ion intensity behind an exit slit was recorded as function of time, while the magnetic field was scanned. Such a spectrometer is easier to operate, but is less sensitive than a spectrograph, since it records at one instant only ions of one specific mass and charge. 


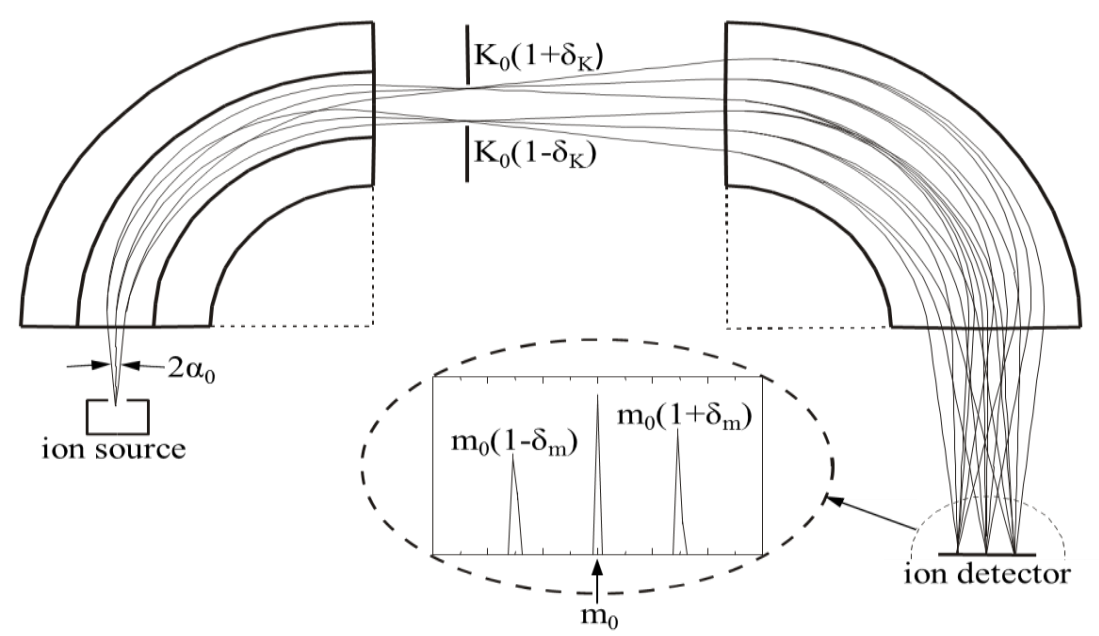

Figure 1. An angle- and energy-focusing mass spectrograph is illustrated that separates equally charged ions of energies $K=K_{0}\left(1 \pm \delta_{\mathrm{K}}\right)$ and masses $m_{0}$ and $m_{0}\left(1 \pm \delta_{m}\right)$. Note that, if ion source and ion detector would be exchanged, the mass spectrograph would still be angle- and energyfocusing [4].

\section{Penning Trap Mass Analyzers}

A very different way to determine the masses of ions of given charge is to record the frequencies $\omega$ of their circular motion in a homogeneous magnetic field $B_{0}$. This $\omega=q B_{0} / \mathrm{m}$ is independent of the initial angles of inclination $\alpha_{0}$ of the ions' trajectories and most importantly of the ions' energies $K$. Ions of larger $K$ simply move along larger radii, but the time to traverse a full circle does not change. This pure mass/charge dispersion due to a physical law allows so-called Penning trap spectrometers to reach very high mass resolving powers though these decrease linearly with increased ion masses.

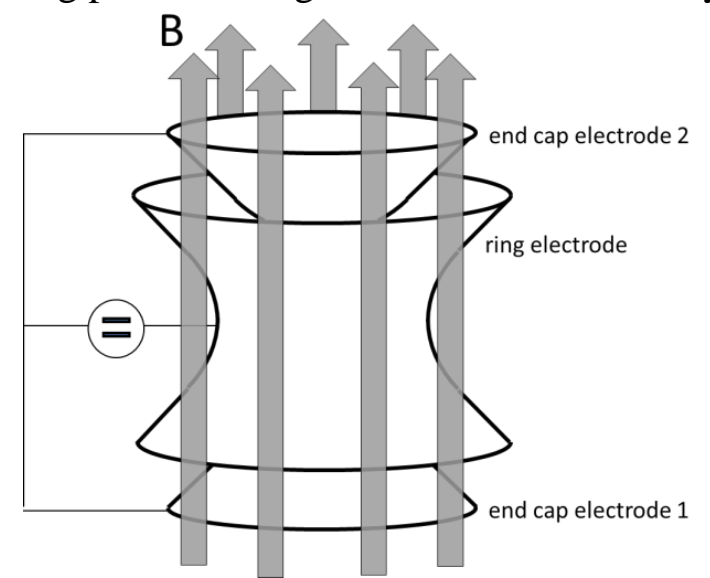

Figure 2 Illustrated is one possible electrode arrangement which, if submersed and aligned in the magnetic field of a Penning trap establishes fields that drive all ions to the magnet midplane [6, 7], if proper DC potentials are applied to the electrodes. If additionally at least one of the shown electrodes is azimuthally divided, the cycling ions induce potentials in these sections, which after a Fourier transformation reveal the frequencies of the cycling ions.

Though the magnetic field confines the ion motion in the plane of deflection, there is no confinement in the perpendicular direction. This can be achieved, however, by establishing a superimposed electrostatic field that drives the ions back to the mid-plane of the magnet $[6,7,8]$. If the correspondding electrodes are sectioned azimuthally, signals induced by the cycling ions are formed in these sections, which, after a Fourier transformation, directly reveal the ion cycling frequencies and thus the masses of the ions. However, it is also possible to establish a high frequency excitation field between some electrode sections, which, depending on the excitation frequency, will cause only ions 
of a corresponding mass/charge ratio to gain energy and so increase the radii of their trajectories. The magnitudes of these radii can be determined, when the ions are extracted from the Penning trap parallel to the magnetic field lines, in which case the azimuthal ion energy is transferred to the kinetic energy of the extracted ions [6]. By using this TOF-ICR technique mass precisions of a few $10^{-9}$ have been achieved, which for ions of masses $\approx 100 \mathrm{u}$ corresponds to a few $\mu u$. Reference masses for such measurements are best obtained [8] from molecular ions that can be produced for all isobars.

\section{Time-of-Flight Mass Analyzers}

The flight times of ions of equal charge $q$, that move through electromagnetic fields as well as through field-free regions depend on their velocities $v=\sqrt{2 K / m}$ and consequently on the ions' masses $m$ and energies $K$. Mono-mass ions of energies $K_{0}\left(1+\delta_{K}\right)$ pass through such systems in times $T_{0}\left[1+(T \mid \delta) \delta_{K}+(T \mid \delta \delta) \delta_{\mathrm{K}}^{2}+(T \mid \delta \delta \delta) \delta_{\mathrm{K}}^{3}+\cdots\right]$ including aberrations to third order in $\delta_{K}$. Designing such systems so that ions of higher energies move along longer flight paths, it can be achieved that at least the higher-order aberrations in $\delta_{K}$ are eliminated [10-17]. In most cases such so-called energy-isochronous time-of-flight mass analyzers are designed to be also angle focusing.

- Determining masses of high energy ions start- and stop-detectors can be placed at the beginning and at the end of such energy-isochronous time-of-flight mass analyzers. Good detectors for high-energy ions consist of thin foils through which the ions must pass releasing on both foil surfaces secondary electrons, which can be recorded as timing signals $[11,13]$.

- For low-energy ions such detectors can only be used as stop detectors, while the start time, is best determined from the timing of ion pulses when extracted from ion-storage devices [13].

\section{a. Sector-Field Time-of-Flight Mass Analyzers}

One way to build an energy isochronous time-of-flight mass analyzer is to use magnetic and/or electric sector fields. Such systems can be designed so that ions of different masses, charges and energies as well as different trajectory inclinations $\alpha$ are laterally focused to the same position. This allows high ion transmissions [10-13], while ion masses are determined from ion flight times.

Obviously the flight time resolving power $T / \Delta T$ in a time-of-flight mass analyzer increases with the length of the flight distance, so that it is advantageous to use a given isochronous flight path repeatedly. This is the case, when the ion flight paths are the ion trajectories in an accelerator storage ring $[11,12]$. The principle of such an isochronous time-of flight mass analyzer is shown in part (a) of Figure 3, illustrating that at a special ring position the ion passage time depends only on the ion masses but not on their energies. Placing a thin foil at this position, the passage of energetic ions is recorded after every lap by monitoring the released secondary electrons. Such timing signals can be recorded for a few hundred laps of an ion, before it is scattered out of the phase space of the ion beam. As long as these ions survive a sufficient number of laps their masses can be determined with mass resolving powers of $R_{m} \geq 200,000$ [13]. There is also a way to achieve high mass resolving powers in a non-isochronous ring, as is illustrated in part (b) of Figure3. In this case the ions interact at every cycle with a beam of monoenergetic quasi parallel beam of electrons, so that at the end they move with substantially the same velocity as the electrons [13,14]. In such systems the ion flight times depend only on the ion masses, in which case the frequency of ion passages can be recorded by Schottky pick up electrodes similarly as in a FTICR-MA achieving mass resolving powers of up to 500,000 [13]. However, this method can only be performed for nuclei that live longer than the ion cooling process, which usually requires several seconds of ion-electron interaction. 

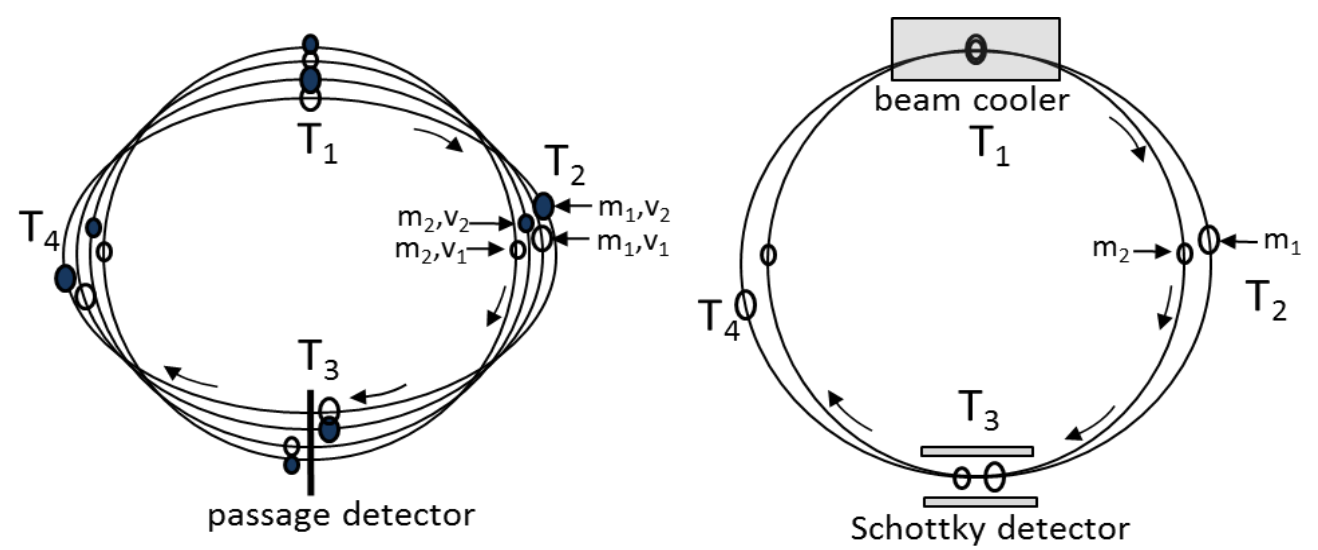

Figure 3 Shown are two principles of precise mass measurements of ions of masses $m_{1}, m_{2}$ in a storage ring. In case (a) the ring is operated isochronot( $(k)$ y, ensuring that ions of velocities $v_{1}, v_{2}$ pass through the passage detector simultaneously at times that depend on the ion masses only. In case (b) the ions interact repeatedly with a beam of electrons of equal velocities, in which case the ions slowly change their velocities to those of the electrons so that their cycling times depend on the ions' masses only.

Isochronous time-of-flight mass analyzers can also be built from arrangements of electrostatic sector fields $[10,15,16]$, provided the ion energies are only a few $\mathrm{keV}$. Such isochronous systems are also able to achieve time-of-flight mass resolving powers $R_{m} \geq 100,000$. Since electrostatic sector fields can easily be switched, it is also easy to enter or extract ions into such a time-of-flight mass analyzer.

\section{b. Mirror-Type Time-of-Flight Mass Analyzers}

Based on the principle that ions of higher energies penetrate deeper into a retarding electric field than ions of lower energies, isochronous time-of-flight mass analyzer can be constructed also. Designing the retarding field [17] as shown in Figure 4 the field strengths $E_{1}$ and $E_{2}$ can be chosen so that the flight time aberrations $(T \mid \delta) \delta_{K}$ and $(T \mid \delta \delta) \delta_{K K}^{2}$ vanish and only smaller even higher-order aberrations re-main. In systems built as shown in Figure 4, the ions must pass through 4 grids before they reach the final ion detector which causes intensity losses of several 10\%. In such systems mass resolving powers of several 10,000 have been reached though the fields close to the grids are not perfect [13].

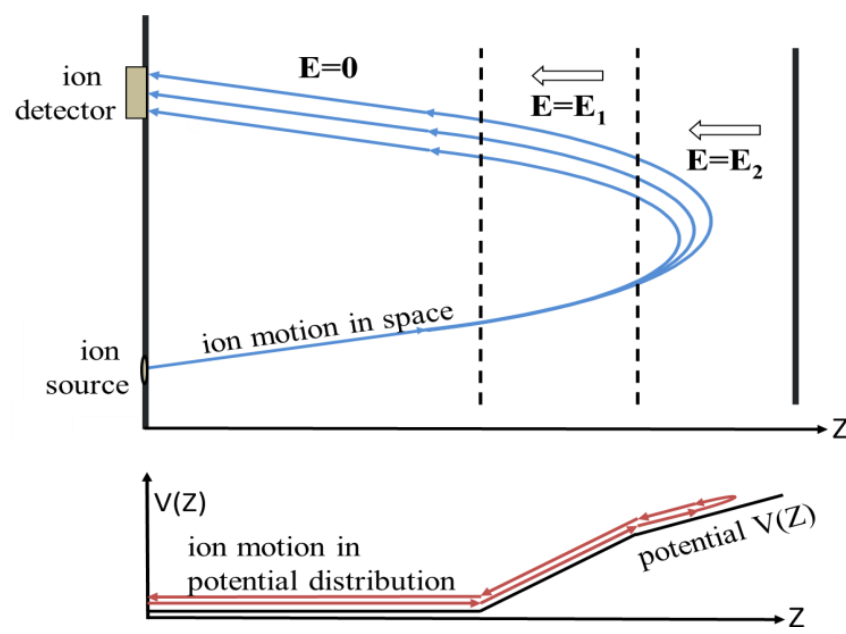

Figure 4 Ion trajectories are shown in a time-of-flight mass analyzer formed by three regions of electric retarding fields separated by grids. In such a system the ion flight time is isochronous to second order, i.e. $(T \mid \delta)=(T \mid \delta \delta)=0$, if $\mathrm{E}_{1}$ and $\mathrm{E}_{2}$ are chosen properly. 
The retarding fields in the mirrors of a time-of-flight mass analyzer can also be formed without grids by metallic rings to which proper potentials are supplied. This requires complex mathematical calculations but achieves good electric fields as well as $100 \%$ ion transmission. The use of grid-free ion mirrors in energy isochronous time-of-flight mass analyzers opens the possibility to arrange several such mirrors in series [18,19] and to use the ion flight path repeatedly as is shown in Figure 5. In such a multi-pass time-of-flight mass analyzer one can introduce the ions through one of the ion mirrors, when its fields are switched off, or by injecting them through an electric field formed between the electrodes A and B. In both cases it is advantageous to accumulate ions for a certain time in some ion trap $[19,20]$ before they are injected into the ion-race-track between the ion mirrors.

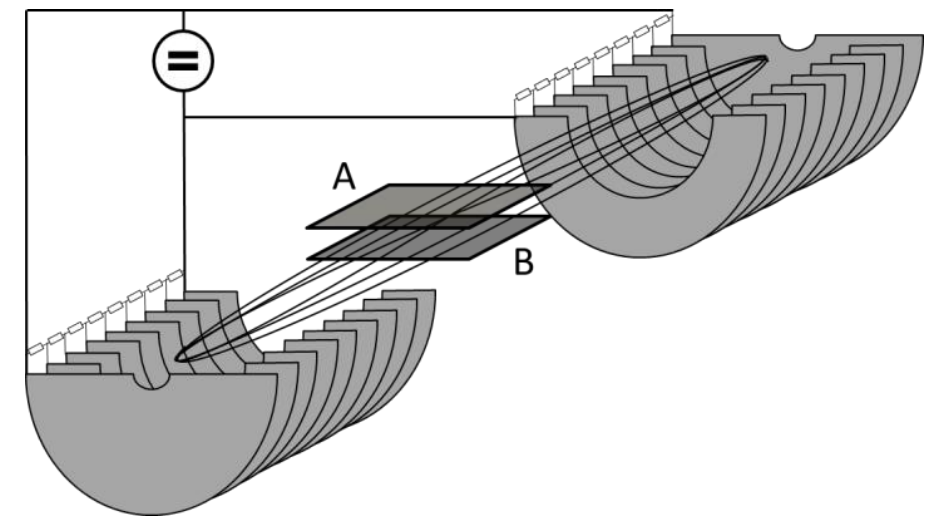

Figure 5 Shown is a multi-reflection time-of-flight mass analyzer, in which the fields in the mirrors are configured so as to achieve energy isochronous ion flight times between the ion mirrors. In detail a short pulse of ions is injected into the system through one of the ions mirrors, when the electric fields in this mirror are switched off or by injecting these ions obliquely between the electrodes A and B, when an electric field is established between them. The overall ion flight times and so their masses can be determined by Fourier transforming the signals induced in the electrodes A and B or by switching off one of the ion mirrors and then record the exiting ions in a classical fast ion detector.

However, the electrodes $\mathrm{A}$ and $\mathrm{B}$ can also deflect undesired ions repeatedly and/or record the induced voltages of passing ions. Alternatively one can record the arrival times of ions downstream of one of the ion mirrors, when its fields are switched off. In this case, however, mass spectra of ions that have performed $\mathrm{N}, \mathrm{N}+1, \mathrm{~N}+2 \ldots$ laps are recorded as overlapped spectra and the overall mass spectrum must be reconstructed by using a mathematical unscrambling procedure. In this way mass spectra have been recorded with mass resolving powers of $\approx 200,000$ [20].

\section{The Orbitrap}

Forming two rotationally symmetric surfaces and arranging them coaxially around a Z-axis (see Figure 6) and applying to them different potentials, properly injected ions can rotate around the inner surface and are still free to move along the Z-axis [21]. Shaping the electrodes as illustrated in Figure 6, two electric ion mirrors are formed in the regions of increased fields caused by smaller electrode distances. Consequently the ions are reflected back and forth between these regions, wherein the flight times between them are energy isochronous. Splitting the outer electrodes as shown in Figure 6 , the repeatedly passing ions between the mirrors induce voltages in the two parts of the outer electro-des, which after a Fourier transformation reveal the ion passage times between the ion mirrors [22].

The most important advantages of an Orbitrap are its small size and its remarkably high mass resolving power [22] of well more than 100,000 for light and for heavy molecular ions. The most important disadvantages are that the construction of an Orbitrap requires to form the inner and outer 
electrodes with high mechanical precision and that the mass analysis of a sample takes about one second and so requires relatively long measuring times.

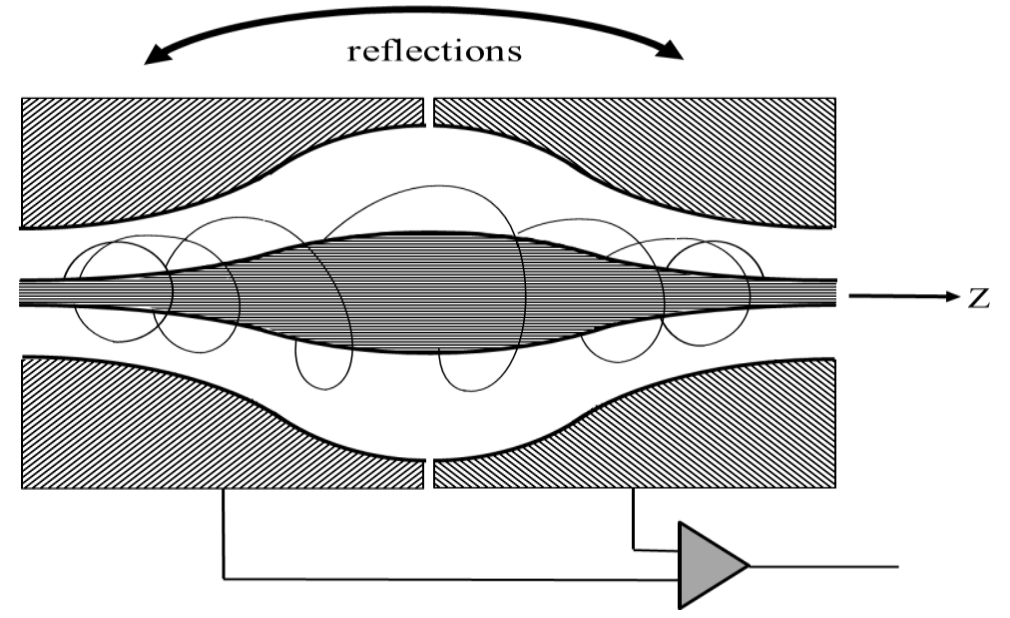

Figure 6 Sketch of an Orbitrap and some ion trajectories within this system. Note that the ions are reflected energy-isochronously between the two regions in which the distance between the inner and outer electrode reduces and the electric fields increase so that they act as mirrors. Note also that the passing ions induce voltages in the two sections of the outer electrode, which after a Fourier transformation reveal the frequencies of the isochronous ion passages.

References:

[1] FW Aston, Philos. Mag. 38 (1919), p. 709.

[2] S Wanjo et al, Astrophys. J. 606 (2004), p. 1057.

[3] SP Gygi et al, Nat. Biotechnol. 17 (1999), p. 994.

[4] H Wollnik in "Optics of Charged Particles", (Acad. Press, Orlando).

[5] AO Nier, Phys. Rev. 50 (1936), p. 1041.

[6] IA Boldin and EN Nikolaev, Rap. Comm. Mass Sp. 25 (2011), p. 122.

[7] G Bollen et al, Nucl Instr. Meth. B 70 (1992), p. 480.

[8] S Eliseev et al, Phys. Rev. Lett. 110 (2013), 082501.

[9] S Naimi et al, Int. J. Mass Spectrom. Ion Phys. 340 (2013), p. 38.

[10] H Wollnik, Nucl. Instrum. Methods 186 (1981), p. 441.

[11] JM Wouters et al, Nucl. Instrum. Meth A 240 (1985), p. 77.

[12] H Wollnik, Nucl. Instrum. Meth. B 26 (1987), p. 267.

[13] H Wollnik, Int. J. Mass Spectrom. 349 (2013), p. 38.

[14] B Franzke, Nucl. Instrum. Meth B 24 (1987), p. 18.

[15] T Sakurai et al, Int. J. Mass Spectrom. 66 (1985), p. 283.

[16] M Nishiguchi et al, J. Mass Spectrom. 44 (2009), p. 594.

[17] BA Mamyrin and VI Karateev, Sov. Phys. JETP-USSR 37 (1973), p. 45.

[18] H Wollnik and M Przewloka, Int. J. Mass Spectrom. 96 (1990), p. 267.

[19] H Wollnik and A Casares, Int. J. Mass Spectrom. 227 (2003), p. 217.

[20] P Schury et al, Nucl. Instrum. Meth. B 317 (2013), p. 537.

[21] KH Kingdon, Phys. Rev. Lett. 21 (1923), p. 408.

[22] AA Makarov, Anal. Chem. 72 (2000) p. 1156; 78 (2006), p. 2113. 